\title{
Correlations between reduced expression of the metastasis suppressor gene KAI-1 and accumulation of p53 in uterine carcinomas and sarcomas
}

Juliane Briese $\cdot$ Heinrich M. Schulte $\cdot$ Maria Sajin •

Christoph Bamberger • Katja Redlin •

Karin Milde-Langosch • Thomas Löning •

Ana-Maria Bamberger

Published online: 5 July 2008

(C) Springer-Verlag 2008

The author's affiliations were given incorrectly. The correct affiliations are given here.

The online version of the original article can be found at http://dx.doi. org/10.1007/s00428-008-0608-7

J. Briese $\cdot$ C. Bamberger $\cdot$ A.-M. Bamberger

Section on Endocrinology and Metabolism of Ageing,

University Clinic Hamburg-Eppendorf,

Hamburg, Germany

J. Briese $(\bowtie)$

Department of Pathology and Tumor Biology,

University of Leeds, St. James's Hospital,

Beckett Street,

LS9 7TF Leeds, UK

e-mail: j.briese@leeds.ac.uk

H. M. Schulte $\cdot$ K. Redlin

Endokrinologikum,

Hamburg, Germany

M. Sajin

Department of Morphopathology,

University of Bucharest,

Bucharest, Romania

K. Milde-Langosch

Department of Gynecology,

University Clinic Hamburg-Eppendorf,

Hamburg, Germany

T. Löning

Department of Pathology, Albertinen-Hospital,

Hamburg, Germany 